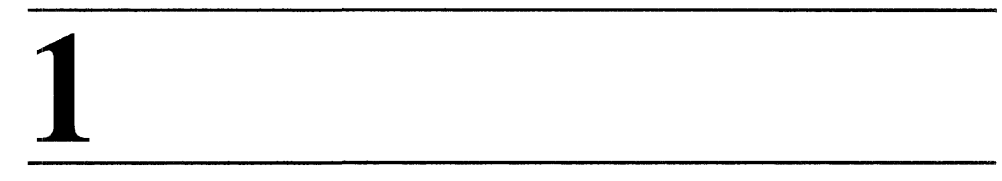

\title{
MINING WAFER FABRICATION: FRAMEWORK AND CHALLENGES
}

\author{
Jemmy Soenjaya, Wynne Hsu, Mong Li Lee, \\ AND TACHYANG LEE
}

\subsection{INTRODUCTION}

The increasing market expansion of electronic devices such as handphones, computers, and television sets has provided the impetus for development of high-tech industry. Wafer fabrication, or the process of producing an integrated circuit on semiconductor materials, plays an important role in manufacturing the fundamental components of electronic devices. Manufacturing is typically a controlled process, where the process flow is carefully and systematically designed. However, random events and subtle changes in environment might cause failures. As with many other manufacturing processes, wafer fabrication often faces fluctuation in products' quality.

In order to detect possible failures, various sensors monitor the process history and record intermediate quality measurements of the production process. The data collected aim to provide insight of the production process, to improve efficiency, and to identify the causes of problems that may during the manufacturing process. While the collection of large quantities of data has been facilitated by low-cost storage devices and automated data collection technologies, there has been relatively little effort made to automate the analysis of the accumulated data. Instead, human experts often analyze selected portions of the data only when problems occur during production or when it is required to optimize the production process. A lot of information regarding the production processes is left unexplored.

Knowledge discovery is described as "the non-trivial extraction of implicit, unknown, and potentially useful information from data" (Frawley et al., 1991).

Next Generation of Data-Mining Applications. Edited by Kantardzic and Zurada ISBN 0-471-65605-4 (C) 2005 the Institute of Electrical and Electronics Engineers, Inc. 
This consists of several steps, one of which is data mining, or automatic pattern extraction from data. Knowledge discovery aims to reduce a large amount of data into a manageable amount of useful knowledge, which can then be processed further by human experts for analysis purposes. Data mining can reduce the time needed to analyze these data as well as provide engineers with valuable insight extracted from the data, which is otherwise unknown.

Analyzing wafer fabrication data poses many challenges. The fabrication process is complex and error-prone. Many factors contribute to fluctuations in the yield obtained. This process utilizes machines with high precision, often measuring up to the nearest micrometer or even nanometer. The entire manufacturing process typically takes weeks or even months to complete, during which wafers go through hundreds of operations generating a huge amount of data to be collected from the various sensors each day. Engineers have to scan these data for relevant information to increase the yield.

Traditionally, engineers use an approach called "Design of Experiment" (Montgomery, 1991) to solve the low-yield problem. This approach consists of a series of experiments whereby several input factors are varied to see how they affect the output of the fabrication process. The key is to be able to formulate the hypothesis as quickly as possible in order to reduce the number of possible factors. This is because the number of experiments is exponential to the number of factors involved. To pinpoint the possible cause of low yield, engineers often have to dissect the faulty wafers to observe the layers of materials added, the patterns formed on its surface, and the graphical view of the faulty patterns. On average, engineers can take weeks or even months to formulate their hypothesis and perform experiments to verify their hypothesis. Furthermore, since a human can only handle a small number of factors at any one time, the large number of operations and thousands of possible factors are simply overwhelming.

In this chapter, we propose a framework that aims to integrate data-mining solutions into the wafer fabrication process to identify causes of failure and low yield. The framework is designed to support the efficient extraction of knowledge from wafer fabrication data, as well as to assist engineers in formulating their hypothesis within a shorter time period.

The rest of the chapter is organized as follows. Section 1.2 gives the background of the wafer fabrication process, and the types of data collected. We also discuss the various issues and challenges involved in mining from wafer fabrication data. Section 1.3 gives a survey of the related work done in mining wafer fabrication data, and the commonly used techniques in supervised and unsupervised learning. Section 1.4 describes the proposed framework, and the details of the preprocessing, defect detection and filtering steps. Section 1.5 shows the results of the experiments performed, and we conclude in Section 1.6.

\subsection{BACKGROUND}

Wafer fabrication is a process of producing integrated circuits using semiconductors. The basic material consists of a disk of silicon called a wafer. Each 

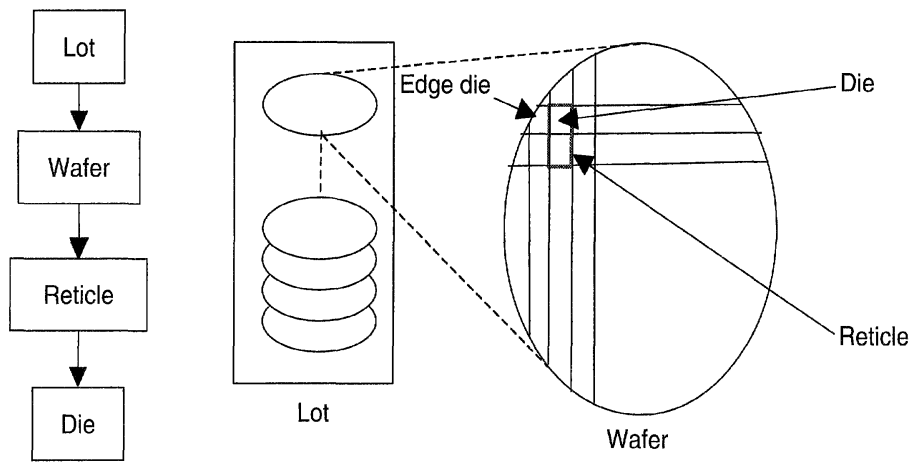

Figure 1.1. A production unit.

type of product has a route, which refers to a sequence of operations that has to be carried out on the raw wafer. Each operation in a route is identified by its operation number. Some of these operations include adding a layer to the wafer, drawing a pattern on the wafer, covering the pattern with a photo layer, and etching the pattern. Each operation is based on a specific recipe, which is the blueprint of what an operation entails. Examples of blueprints are the thickness of a layer to be added, or the pattern to be drawn on the wafer. An operation consists of several scripts, and each script consists of several steps. However, a script is seldom used to identify a particular operation. Instead, a unique identifier $\langle$ route, operationno, step $\rangle$ is used for the process step. Some examples of process steps include "wafer enters a machine," "addition of a layer to a wafer," "wafer exits from a machine," and so on.

Figure 1.1 shows a unit of production. The largest unit is a lot, which is a stack of wafers. A wafer is a semiconductor disk, on which the integrated circuit is made. All the wafers in a lot go through the same route, and they undergo the same operations based on the same recipe. In general, a lot consists of 25 wafers. Note that in some situations a lot may consist of less than 25 wafers. This is because the engineers may take some wafers out for testing purposes so as to improve the yield, or to trace the cause of a yield drop. In this case the lot is split into several lots, and the resulting lots are called child lots.

A wafer disk is segmented into small rectangles, called dies, which are the smallest unit. A group of dies forms a functional unit called a reticle. A functional test structure is built at the boundary of each reticle for testing purposes. The size of a die and a reticle varies from product to product. Each product has its standard die count, which is the total number of dies a wafer should contain. The actual die count of a wafer is obtained during the final test.

\subsubsection{Operational Data}

Throughout the fabrication process, various types of data are collected, from process history, intermediate measurements, to final testing. Operational data are 
organized on a daily basis, recording all the operations that are carried out on that day together with the intermediate measurements, the machine's maintenance, the name of the operator performing the task, and other related data. There are two main operational data that we focus on: the process history, which is also called the lot history, and intermediate measurements, which is called metrology. Both lot history and metrology use lots as basic unit, since all the wafers in a lot undergo the same operations and stay together throughout fabrication process, unless a split is performed.

Lot history captures the operations executed on the lots in a particular day. Among other things, it records the date and time the operation was performed, the machine where the operation was performed, which has an event name and a process number, the lots' identification number (id), recipe, product name, and route used. In our analysis, all the wafers originate from the same product, and thus product name and route is inconsequential. The lot history uses the combined date and time as the main key for its relation table, and it uses event name and process number as the secondary key.

The second operational data is metrology. The metrology records intermediate measurements taken as part of process control. If a wafer's measurements differ from the specifications, then the wafer may lead to a lower yield. Some examples of these measurements are the thickness of added layer, the size of patterns drawn on the surface, and the depth of layer etched. Although process control is important in monitoring the state of the wafers throughout the fabrication process, it is also expensive in terms of required time and manpower. Too many measurements result in a slower fabrication process and a lower production throughput. Thus, measurements are done only after important steps as specified by the engineers. Furthermore, only some of the wafers in a lot are measured, on which randomly selected points are measured. The parameters of the measurements state how many wafers per lot and how many points per wafer should be measured. The lot id is not recorded in this table. The parameter also specifies the type of measurements - for example, the length of pattern in $x$ dimension, or $y$ dimension, or average length of both. The key of this relation is similar to lot history - that is, date and time as primary key, and event name and process number as secondary key.

\subsubsection{Final Tests Data}

In addition to operational data, there are also final tests data. The purpose of the final test is to ensure that all the dies/chips produced function as specified. There are two tests that are performed, a functional level test called the parametric test, and die level test. The parametric test evaluates the test structure of the reticle, which consists of hundreds of electrical characteristics tests. There are five sites (or reticles) tested for each wafer. The second test is performed at die level, where a probe wafer is used to label each die of the wafer. The labeling process is called binning. The bin number goes from 0 to a certain number as defined by engineers. Bin 1 denotes that the die is working properly. All other bin numbers 
denote some types of error/failure characteristic. At the end of this die level test, a wafermap is obtained, which is the graphical representation of the wafer with each die labeled by a bin number. The yield of the wafer is then defined as the number of dies that fall into bin 1 over standard die count. Both parametric and die level tests are performed on all wafers because it is important to package only the properly working chips.

\subsubsection{Issues and Challenges}

Some of the issues and challenges that affect the design and choice of algorithm in mining wafer fabrication data are as follows:

1. Data Granularity. Two different types of data are involved, namely, the operational data and the final tests data. Although these two types of data capture different aspects of the fabrication process, they are interrelated. However, there is a gap between these two types of data. The operational data are lot-based, while final test data are wafer-based. Thus, we need a way to bridge this difference in data granularity.

2. High-Dimensional Data with Small Sample Size. Operational data provide details on fabrication process. They contain various process parameters and intermediate measurements that can be used to trace the cause of the problem or to find ways to optimize fabrication output. These data contain long sequences and a small sample size. Mining high-dimensional data with very small sample size is inherently difficult. To overcome this problem, we need to reduce the dimensionality by eliminating parts of the sequence that are irrelevant.

3. Large Number of Low-dimensional Data Points. Final test data reveal the output of the fabrication process, from which we can assess the rate of success of the fabrication process by observing the yield obtained. This data are also the main source for identifying the existence of problems that arise in fabrication. The final test data have a huge number of points in relatively small dimensions. This requires us to design an algorithm that is efficient in terms of the number of points processed. Since the data are not labeled, the algorithm to extract pattern should be of the unsupervised class to group similar failure signature together.

4. Skewed Data. Both operational and final test data are highly skewed, since we can only obtain a small sample of problematic wafers or lots. The problem is usually short-lived; that is, once the problem is solved, we do not expect to see it reappearing again in the near future. Thus, an algorithm that is able to mine information from a small sample size is important. In addition, we also require the algorithm to be not only accurate, but also sensitive in detecting the problematic wafers. For example, if $80 \%$ of the wafers is normal, then an algorithm that outputs $100 \%$ of the wafers to be normal with a relatively high accuracy of $80 \%$ is not sensitive enough since it has failed to identify the existence of problematic wafers. 


\subsection{RELATED WORK}

In this section, we discuss the existing methods to mine wafer fabrication data. The main approaches to supervised and unsupervised learning are also highlighted.

\subsubsection{Mining Wafer Fabrication Data}

Mining wafer fabrication data has generated a lot of interests because of its unique characteristics and the possible benefits gained. François and Ives (1999) advocated that data analysis can help discover causes of yield drop. They successfully identified the cause of a manufacturing problem using statistical techniques for data analysis such as ANOVA (Devore, 1995) and regression techniques. Coupled with the engineers' expertise, they demonstrated that data analysis can be a valuable tool in improving a wafer fabrication's yield.

Bertino et al. (1999) reported their work on wafer fabrication data. They applied two data mining tools, namely, Mineset (Brunk et al., 1997) and Q-Yield (Quadrillon, 2003). Based on their experience, they found that general datamining approaches do not reveal much of the causes of failures. They highlighted the difficulties in using the association rule from Mineset-namely, that the minimum support and confidence used is inappropriate. In fact, it is necessary to set the minimum support to be very low because the number of problematic wafers is typically low. However, a low minimum support leads to an explosion in the number of frequent itemset candidates. To overcome this problem, they constructed an interest graph of factors that engineers are interested in. A general algorithm was designed to traverse this interest graph and to produce the hypothesis. While the interest graph also served as a filter to find the factors that may contribute to the low yield, the number of factors that needs to be checked remains exponential. Furthermore, the engineers are required to supply the interest graph, as well as to label each step of operational data to indicate if the process succeeded or failed.

Given that the semiconductor industry faces increasing product design complexity, and a decreasing window period to manufacture their products, it is becoming critical to identify factors that contribute to product defects. Tobin et al. (1999) presented techniques that can efficiently identify the spatial signature of problematic wafers. Wafers with similar spatial signature are grouped together. The cause of a defect can be deduced by examining the causes of previously stored wafers with similar problem signature. The signature can also be associated with one or more process steps to provide insight on the possible causes. For example, tightly clustered objects can be correlated with insufficient etching. Their results showed that wafer defects can be recognized based on their spatial signature. A technique that is able to reflect on past experiences for solving similar problems in future is also proposed.

Kittler and Wang (2000) explored several data-mining algorithms on wafer data, and they compared the results to the traditional statistical test approach. 
In particular, they compared decision trees to statistical methods such as regression, ANOVA, and Kruskal-Wallis tests. They concluded that decision trees can perform as well as traditional statistical tests. However, the existence of large amounts of missing values may prevent useful analysis to be done. They also noted that the difference between statistical methods and data-mining techniques is that statistical methods typically assume a model; for example, ANOVA assumes that the underlying data has a normal distribution, and thus the result depends very much on whether the right model has been chosen. In contrast, most data-mining algorithms are nonparametric, that is, they do not make any assumptions about the data. However, one has to be careful in choosing the algorithm, because each algorithm has its own bias; for example, the association rule favors common items by having minimum support as one of its criteria.

Another data-mining case study on wafer manufacturing data is reported by Gardner and Bieker (2000). A total of three case studies were presented in the article. The techniques used are Self-Organizing Map/SOM (Kohonen, 1995)-based clustering and rule induction from clusters, embodied in a software tool named CorDex. Sets of data that have been handpicked by the engineers are presented to the system, which will cluster the data. The clusters are labeled according their yield, poor, medium, and high. Rule induction is used to extract discriminating rules that explain the cluster, which are then presented to the engineers. The system is able to find the clusters that represent the failure cases and extract relevant rules for all three case studies. However, the system also generates more clusters than is necessary, and it is unclear how a rule is selected from the list of rules presented. Another drawback of this approach is that the data must be first preprocessed by the engineers. As such, it does not provide for automatic integration to the manufacturing system. However, they have shown that data-mining techniques can be successfully applied to wafer manufacturing application.

Fountain et al. (2000) proposed a decision theoretic approach to construct efficient test procedures, thus reducing the number of dies that need to be tested at the die level test. However, since a mature wafer fabrication tends to have a more stable yield, an influence diagram and utility model can be developed to model the pattern of the output. This model can be used to predict the die pattern of the wafermap-that is, which die is likely to fail the test, how many dies need to be tested, and so on. The system gains by saving resources required by picking dies in a certain order to be tested, based on the model learnt from historical data, rather than testing all the dies. For a normal wafer, the number of dies tested will be small, and thus a high number of dies tested by this system indicates a possible failure. However, the system stops at identifying the existence of failure.

Kusiak (2000) explored yet another aspect of mining wafer fabrication data. He presented a way to decompose the huge amount of data into smaller relations, which can then be processed in parallel. Two possible ways to decompose the data are presented, namely, object-set-wise and feature-set-wise. A rule-based system was also deployed in an industrial case study. The benefits of this approach is that it reduces the time complexity of data-mining algorithms and selects only the relevant features for a particular segment/group. 
All the previous works on wafer fabrication data addresses different aspects of applying data-mining techniques to analyze the data. There is a lack of framework that unifies these solutions into an automated data analysis that can be integrated into wafer fabrication.

\subsubsection{Unsupervised Learning}

A quick survey of data-mining algorithms reveals two big classes of algorithms: supervised and unsupervised. Supervised algorithms learn from labeled data, where each example has its class label, which is the output of the target function that we would like to find out. For example, in weather dataset, the class label can be sunny, cloudy, or raining. The task is then to find the structure of each of the class; in most cases, it aims to predict the label of future unlabeled examples. On the other hand, unsupervised algorithms learn from unlabeled data. The task is to find pattern, structure, or regularities that exist in the data, such as groups of similar examples.

Clustering is the most commonly used unsupervised algorithm. The aim of clustering is to discover clusters or groups of similar instances in the data. In general, the data are represented as points in multidimensional space. Each dimension corresponds to a feature of the data (columns in relational table). The task is then to group points that are close/similar to each other into a group, such that points that belong to a group are closer to each other compared to their distance to points that belong to another group. The definition of closeness or similarity (or distance and dissimilarity) often varies from one domain to another. Distance metrics such as Euclidean (squared difference) or Manhattan (absolute difference) is often used in geometrical applications, while Kullback-Leibler distance (Kullback and Leibler, 1951) and its variances are often used for color histogram and distributions of data.

Clustering techniques have been studied under various contexts and used in many disciplines. Jain et al. (1999) present an overview of clustering algorithms. There are two prominent classes: hierarchical and partitional algorithms. Hierarchical algorithms cluster $n$ points in $n-1$ steps, where at each step either two clusters are merged (bottom up, agglomerative) or one of the clusters is divided into two (top down, divisive). It produces a dendogram where the leaves represent the data points. Figure 1.2 shows an example of a dendogram.

The most popular partitional algorithm is the $k$-means (Lloyd, 1982; MacQueen, 1967; Linde et al., 1980). $K$-means divides the dataset into $k$ distinct clusters, where $k$ is supplied by the user. $K$-means works by first randomly selecting $k$ representative points as the center of the cluster and then iteratively refining these points by moving the cluster's representative to the center of gravity of points that belong to that cluster - that is, points that are closer to this representative point as compared to the other $k-1$ points. It can be proven that this algorithm will converge to local minima that minimize the sum of squared error between the points and their respective representative clusters. However, these local minima can be far from the global optimum. This algorithm is sensitive to 


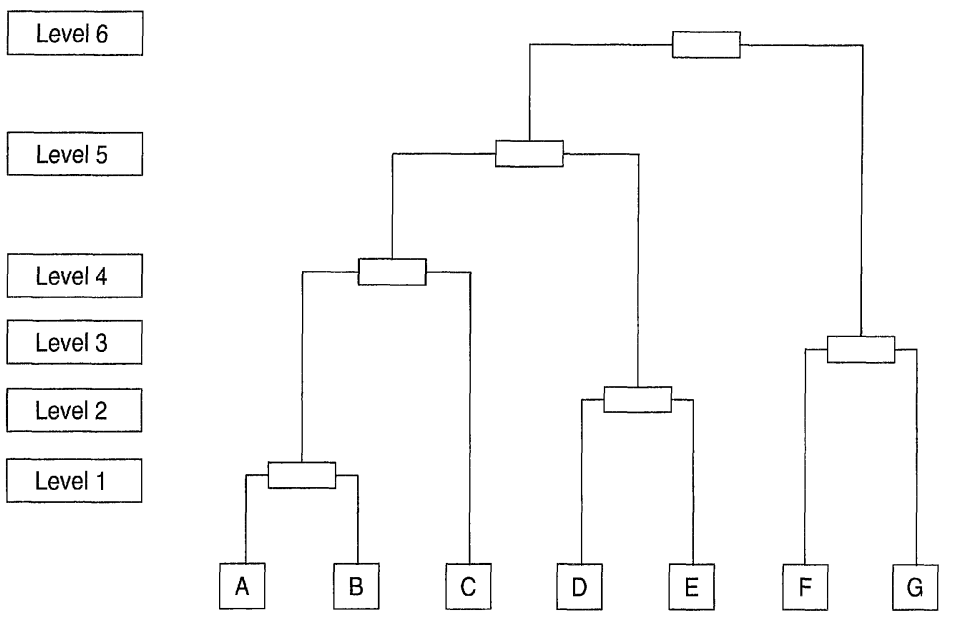

Figure 1.2. Example of a dendogram.

the starting $k$ points chosen. Bradley et al. (1998a) present a way to choose a better set of $k$ points by severely under-sampling the data.

Dubes and Jain (1988) study these two approaches of clustering and conclude that hierarchical clustering is superior to $k$-means in most cases. However, the cost of performing hierarchical agglomerative clustering (a bottom up hierarchical clustering) on $n$ points of $d$-dimensional data is $O\left(d n^{3}\right)$ when a dissimilarity matrix is used and the fastest algorithm runs at $O\left(d n^{2} \log n\right)$ by using a priority queue. $K$-means algorithm runs on $O(n k t d)$, where $k$ is the number of cluster desired and $t$ is the number of iterations until the algorithm converges. Since $k$ and $t$ are much smaller than $n, k$-means runs linear in the number of data points. When $n$ is large, the cost of performing hierarchical algorithm is too expensive. Bradley et al. (1998b) proposed a single-scan framework for clustering large databases.

Besides hierarchical clustering and $k$-means, clustering techniques such as density-based DBScan (Ester et al., 1996) and representatives-based CURE (Guha et al., 1998) make use of statistical properties of the database to generate clusters. Zhang et al. (1996) present a BIRCH that uses only one scan of the database to build the clusters under a small memory constraint.

There are also other works to discover regularities on temporal data. These techniques are used mainly in business applications, such as stock price, and in scientific experiments that measure changes over time, such as microarray data in bioinformatics. Regression-based methods such as auto-regression coupled with moving average are often used. Another alternative is a segmented line as used by Povinelli (1999).

For wafer fabrication data, we utilize a hybrid of $k$-means and hierarchical clustering techniques to achieve a tradeoff between speed and accuracy for a large number of points. We also employ segmented-line time-series analysis to filter out irrelevant features. 


\subsubsection{Supervised Learning and Feature Selection}

Many methods have been proposed to learn from labeled examples, the majority of which are derived from the machine learning field. The objective is to learn a function that maps a set of features to a value. Techniques such as Bayesian Learning (Bayesian Belief Network or the simpler Naïve Bayesian), Neural Network, and Reinforcement Learning are discussed in Mitchell (1997).

Supervised learning algorithms aim to predict future unseen cases. This is referred to as classification. The classifier CBA (Liu et al., 1998) is based on association rules (Agrawal and Srikant, 1994), while C4.5 (Quinlan, 1992) is based on decision trees. When the number of features is large, some of these classifiers do not scale well. Furthermore, the robustness of the results is affected when the number of available data points is small.

This leads to the research on feature selection that addresses the problem of selecting relevant features for a particular problem. Various relevance definitions are defined by John et al. (1994), which labels the relevance of features based on the probability of output function. There are two types of feature selection algorithms: wrappers, which wrap around the classifier/learner, and filters, which are independent of the learner used. Tavalera (2000) presented a feature selection based on COBWEB's salience, which can be interpreted as how knowing the cluster label of this point can increase our belief that a feature of this point has a certain value. This is similar to the one that is defined by John et al. (1994). Dash and Liu (1997) presented various categories of feature selection algorithms.

Although there is a general consensus that a relevant feature must be somewhat correlated to the output that we would like to predict, the actual feature selection itself differs from one domain to another. The general guideline is correlation. In this work, we make use of domain knowledge coupled with correlation measures to design a filter for selecting the relevant features.

\subsection{PROPOSED FRAMEWORK}

In this section we propose a framework that serves as a glue to unify the various approaches to mining wafer manufacturing data. Figure 1.3 shows the overall framework. We separate the data into two parts-operational data and final test data-since they are used for different purposes. The operational data provide information required to identify the cause of the problem, while the final test data provide insight about observable problem signature.

Data preprocessing is an important step to convert the raw data collected from production process into a form suitable for mining. Different representations of the data can produce different results. Furthermore, data preprocessing also consolidates data that are scattered over several databases.

Defect detection plays an important role in discovering problem signature as it automatically labels the type of defect from final test. The simplest form of defect detection is thresholding, where "problematic" refers to those wafers whose yield is lower than a certain threshold. Here, we cluster the bin profiles to 


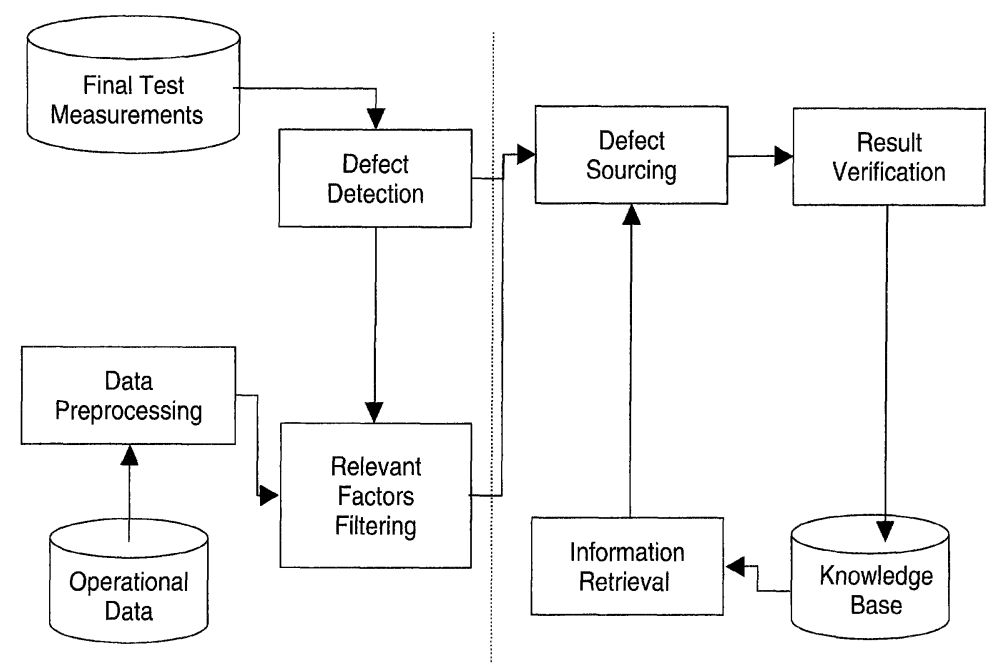

Figure 1.3. Schematic view of mining framework.

detect problem signature. It is reasonable to assume that if the problem signatures are similar, then it implies that the wafers share a similar problem cause.

Wafer manufacturing is a complex process that includes hundreds of steps. As such, a filtering step is required to eliminate irrelevant factors before proceeding to find the pattern from the data and determine the cause of the problem. Here, we utilize the domain knowledge and design a filter-type feature reduction technique. At the same time, we also use the feedback from defect detection to guide us in the removal of irrelevant features.

The right side of the framework in Figure 1.3 consists of a cycle of defect sourcing, verification, information retrieval, and knowledge base. In our framework, once the defect sourcing produces a hypothesis on the cause of the problem, the engineers can verify it by performing experiments and physical examinations of the wafers. A verified solution is subsequently added to the knowledge base for future reference. Details of each components of the framework are given below.

\subsubsection{Data Preprocessing}

There are three types of data in wafer fabrication: lot history, metrology, and die level test results. Lot history and metrology are operational data, while die level test results are the final test data.

Lot history and metrology data are stored daily in separate files. Each day, a list of operations performed, including intermediate measurements taken, are stored in the lot history. Each operation contains date and time, lot id, machine/equipment id, recipe, event name, process, step number, and so on. If the event is an intermediate measurement, then the results of the measurements are stored in metrology. The entries in metrology do not include the lot id of the wafers examined. Thus, 
we have to refer to the lot history to find the corresponding entry and fill in the lot id. In order to integrate the data in these two types of files, we use date and time as the primary key. Event name is used to resolve any entries with the same date and time. As the files are stored on a daily basis, we only search the entries of lot history from the same day as the one from metrology. Once we have combined the two files into a daily report, we group the subsequent processes and measurements into a single entry.

Next, we reformat the list into two types of sequences. The first sequence is based on the lots, where we the list of machines each lot went through and the measurements taken, if any. The second list is based on the machine and process number, which contains the lots that went through this machine, and intermediate measurements taken after this process, if any. Both lists are sorted based on date and time of the process performed.

The final test data that we obtained consist of wafermaps taken from die level tests. Instead of using spatial signature detection, we propose to use the bin profile as the problem signature. A bin profile is the distribution of dies that fall into the various bins as defined by the engineers. These bins represent different types of error/failure found during die level test. The exception is bin 1, which denotes that the die has passed the test. In order to obtain the bin profile from the wafermaps, we count the number of dies that falls into a particular bin.

Figure 1.4 shows an example of a wafer that contains 29 dies and four types of bins. The profile is normalized by total die count. For the product data that was given to us, the standard total die count is 2499. Sometimes the wafermap contains less than 2499 dies. This is mostly due to "zero yield," which is a condition whereby the yield of the wafer is so low that the test is only performed partially. To cater for this case, we add an additional special bin, which represents this set of untested dies. Note that Toblin et al. (1999) did not include the bin label of failed wafers; and, as such, our approach can act as a complement to their method.

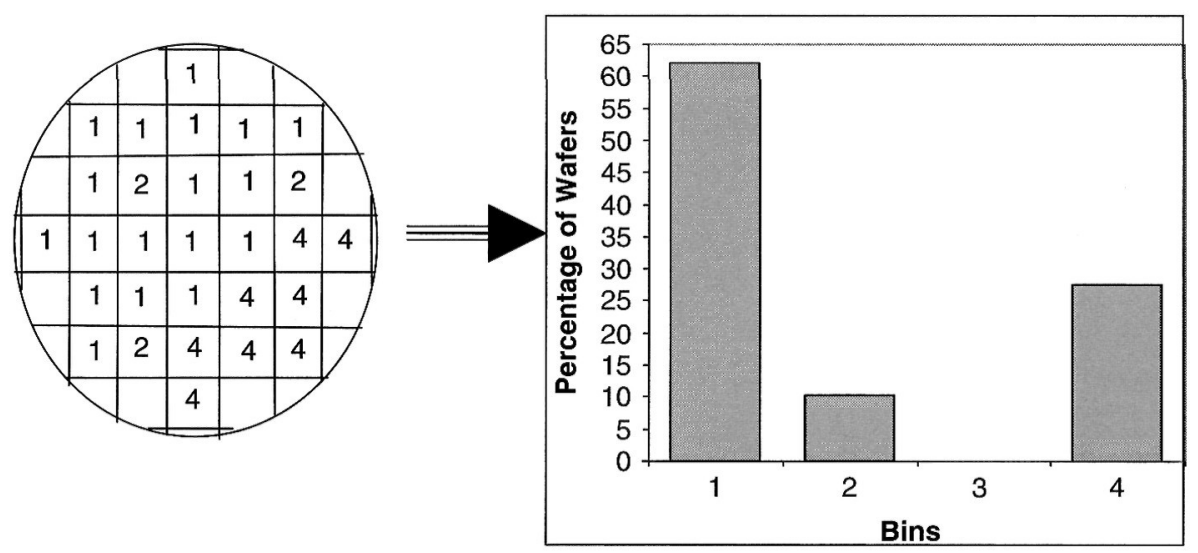

Figure 1.4. Conversion from wafermap to bin profile. 


\subsubsection{Defect Detection}

Automatic defect detection is performed on the final test data. We obtained the production data for the first four months of year 2002 from STMicroelectronics. The die level test data consist of 6490 wafers organized into 286 lots. The bin profile has 15 dimensions, where all the bins other than bin 1 represent error types. We cluster these bin profiles to find wafers that have similar failure characteristics.

Although it has been shown that hierarchical clustering is superior to $k$ means (Dubes and Jain, 1988), its cost is much higher than that of $k$-means. Dash et al. (2001) address this high complexity and propose a method to segment the original space into $p$ partitions with overlapping area $\delta$ between partitions. The parameter $\delta$ is taken from the observation that they refer to as $90-10$ phenomenon. They observe that the lower portion of the dendogram mainly contain points that are close to each other. After a certain number of iterations, the intercluster distance will begin to increase rapidly. $\delta$ is then set to the this critical value when the distance starts to increase sharply. In effect, this method assumes that for the majority of the points, it is adequate to search the closest point locally within a certain area of neighborhood, and the overlapping $\delta$ area allows points from different parts to be combined. Dash et al. (2001) noted that in $90 \%$ of the steps, the intercluster distances are very small. When the distances are very small, it is not possible to differentiate small "inexactness" in the solution. This allows us to use a faster but less accurate method for these $90 \%$ of the points-that is, $k$-means.

However, it is well known that $k$-means might arrive at a local minimum that is far away from the global minimum. Matoušek (2000) presents an asymptotically efficient $O\left(n \log ^{k} n\right)$ time algorithm that approximates the distortion of the result as close as $(1+\varepsilon)$ times the optimal distortion. However, the run time is expensive when the number of dimensions, $d$, and the number of clusters needed, $k$, are large. There are many others that work on this problem. Among them, Bradley et al. (1998a) propose a way to select a good initial start point by subsampling the points, and then they cluster these samples to estimate the start point. These samples will reveal where the means are since the area near the means will have more points. This increases the likelihood that a sample will be picked from that area.

Based on these ideas, we propose to first sample the data and then use these samples as the starting cluster points for $k$-means. Once we have run the $k$-means, we perform a hierarchical clustering on the remaining $k$ clusters by treating each cluster as a point. Note that uniform sampling does not apply in this situation. Instead, we propose a sampling method that takes into consideration the size of the bounding box of the group. Here, the number of points taken for the $i$ th group is $\alpha n d_{i} / D$, where $0<\alpha<1$ is the fraction of points kept, $d_{i}$ is the size of bounding box of the $i$ th group, and $D=\sum_{i=1}^{i=Z} d_{i}$, where $Z$ is number of groups.

Note that this sampling approach is area-sensitive, since it will include more points in a sparse area than in a denser area. Note that a dense area will have a smaller bounding box since the points are closer to each other. In this way, we can prevent points that are far away to be grouped by $k$-means. 
Until now, we have assumed that there is a way to order the points and efficiently separate them into groups of equal sizes. Sorting and splicing can be easily carried out for one-dimensional data. However, for high-dimensional data, we have to assign weights to each dimension to reflect its importance. An alternative is to employ an algorithm such as BIRCH (Zhang et al., 1996) to provide the initial grouping. Here, we propose to use the Principal Component Analysis (PCA) proposed by Hotelling (1993) to map the original axis into principal components, which are orthogonal to each other, that is, there is no correlation between the principal components, which can be ranked based on the variance. Given the orthogonal property of the principal components, we can keep only the first few principal components that have large variances, and we transform our data into these selected components. Figure 1.5 shows an example of PCA where the dots represent the data points and the blue line represents the first principal components.

We observe that if we store the data using this new axis, we will lose the least amount of information. In the same manner, we can reduce the data that we are going to cluster to one-dimensional data by applying PCA and retaining only the first principal component. The data are then sorted, and they are divided into groups of equal size. While we cannot guarantee that we have performed the sorting on the right axis, we are assured that the reduced axis has the most variances. Thus, we can think of it as being the representative of the real distances between points.

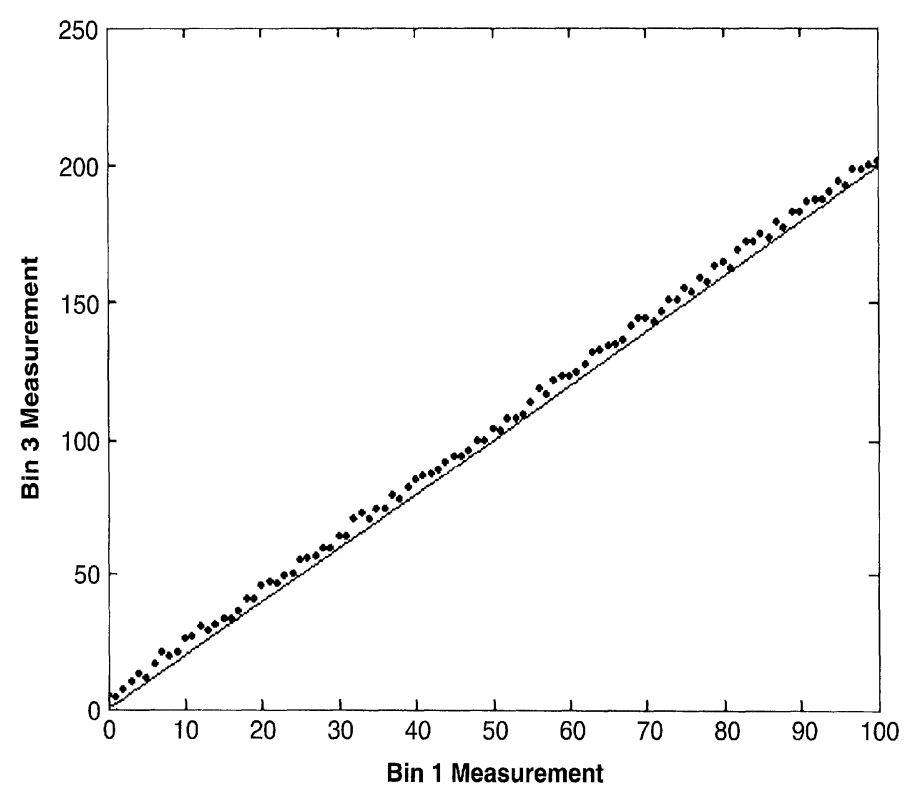

Figure 1.5. An example of PCA. 
Another factor that affects cluster accuracy is that $k$ should be some reasonably large number, such as $10 \%$ of the original number of points. This will allow more flexibility in selecting the initial start points, because each group can have more than one representative point.

\subsubsection{Relevant Factors Filtering}

Having clustered the bin profiles to identify problem signatures, we need to establish a link from the operational data to the problem signatures in order to derive possible causes of defects. However, when we examine the operational data, we realize that we have about 286 lots, each of which is a sequence of length 570 of unique machine id and measurements combinations. These are high-dimensional data, with very few data points.

To enable meaningful mining, we need to eliminate irrelevant features. In supervised learning, we can make use of the label to evaluate the fitness of a feature, such as to select minimum set of features that will result in good predictive performance. Dash (2002) propose the use of entropy to measure the regularity of a feature. Tavalera (2000) present a modified COBWEB's variable utility function to select feature that is more prominent in one cluster but not in other clusters.

Besides reducing the dimensionality of the data, we also need to bridge the granularity gap from wafer-based die level test to lot-based operational data. We do this by representing each lot as a series of $n$ weights: $w_{1}, \ldots, w_{n}$ such that $w_{i}=l_{i} / z$, where $l_{i}$ is the number of wafers that a lot belongs to in cluster $i$, $z$ is the number of wafers in a lot, and $n$ is the total number of clusters. The exception is in the clusters with high yield. Generally, there is only one good cluster. This is because the distance between the good wafers are small, and thus the good wafers will be naturally grouped together into a single cluster. This cluster is also the biggest cluster among all, because there are only a few wafers that experience a problem. As such, we require that a lot must have either $100 \%$ or $0 \%$ weight of a good cluster. If a lot has one wafer that belongs to a cluster which is not a good cluster, then the lot has $0 \%$ weight of good cluster. This is equivalent to saying that if a lot has one wafer that has problem, then the whole lot is labeled as problematic.

We employ two methods to help pinpoint the source of defects. The first method is to establish the expected behavior, any deviation from the expected behavior constitutes probable source of defects. We call this test the variability test. Suppose we have the measurements of wafers that went through a machine. If we transform them into time-series data, then the expected behavior of the machine is shown in Figure 1.6, where the performance of the machine is initially good, degrades over time, and then returns to its original state once maintenance is carried out. If we replace each point with the average of the value of previous $x$ points, or the moving average, then we obtain the graph as shown on the righthand side of Figure 1.6. We observe that there is little fluctuation in the expected behavior. In fact, once the graph is smoothed, most of the values will fluctuate slightly around a value and show a periodic pattern. 

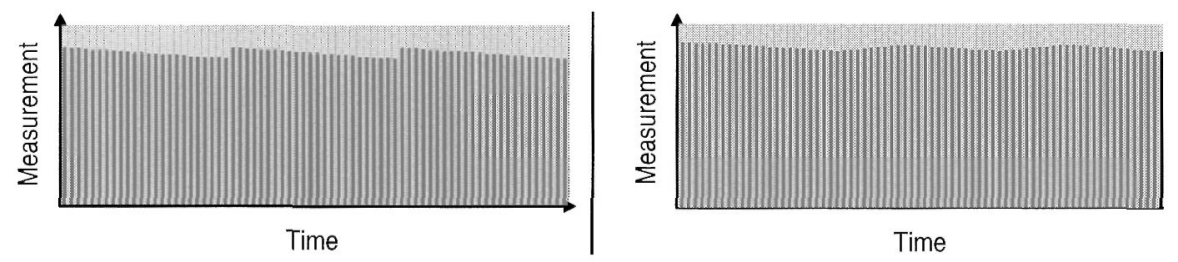

Figure 1.6. Ideal machine measurements: original and smoothened.

To find the deviation from the expected behavior, we first smooth the values of the measurements and find the change points, which are the local maxima and minima. We measure the number of local maxima and local minima and the amplitude difference in a sequence. Once we have found the local optima, we represent the sequence as segmented line. The squared error between the original value and the segmented line representation is calculated, along with the average fluctuations of the amplitude. Using this value, we rank the machines. The machines with a high squared error are likely to be the causes of the defects.

The second method to pinpoint the source of defects is to select the machines or measurements that are able to differentiate the bad lots from the good lots. Here we use the good cluster as the basis of comparison. The intermediate measurements of these high-yield lots are used as the ideal values. Based on this rationale, we collect the measurements for each cluster. The contribution of a lot is based on its weight of the cluster as mentioned above. We then test the difference between the distributions of a cluster and the high yield cluster. If there is a significant difference, then we mark this machine, along with the corresponding measurements and step number, as a probable cause. If there is no significant difference, then we can discard this machine because we cannot tell the difference in quality between low-yield clusters and high-yield clusters by observing the results of this machine. There are statistical tests available for testing the difference between two distributions, such as the $T$-test and the Wilcoxon Rank-Sum Test (Devore, 1995). The $T$-test assumes that both distributions are normal, while the Wilcoxon Rank-Sum Test is a nonparametric test that does not assume the underlying distribution of the data. Here, we adopt the Wilcoxon Rank-Sum Test. The degree of difference is taken from the difference of observed values obtained from the data and the critical value of Wilcoxon Rank-Sum Test. The higher the difference between the two values, the more likely the two distributions are actually two distinct distributions. The machines, along with the corresponding measurements and process number, are ranked based on the degree of difference that they exhibit.

\subsection{EXPERIMENTAL STUDY}

We develop a prototype of the wafer fabrication mining system for initial exploration. The algorithms are implemented in Java, while the data preprocessing is 
TABLE 1.1. Number of Points and Their Corresponding Run Time

\begin{tabular}{cc}
\hline Number of Points & Run Time (seconds) \\
\hline $325(5 \%)$ & $2,395.44$ \\
$649(10 \%)$ & $12,453.28$ \\
$974(15 \%)$ & $24,922.63$ \\
$1,298(20 \%)$ & $51,112.13$ \\
$1,623(25 \%)$ & N.A. \\
$1,947(30 \%)$ & N.A. \\
$2,272(35 \%)$ & N.A. \\
$2,596(40 \%)$ & N.A. \\
\hline
\end{tabular}

${ }^{a}$ N.A., not available.

done by combination of perl and bash scripts. Our programming environment is a unix server Sunfire and linux based cluster connected via GigaEthernet as well as Myrinet. The principal component analysis is performed on a Windows-based machine using MATLAB.

We obtain 4 months of wafer fabrication data from STMicroelectronics that is known to contain problems. The engineers have already performed independent investigations on the cause of the problem in this data. Their findings, however, is not revealed to us until we obtain our results from our experiments.

The size of the raw data is approximately $70 \mathrm{~GB}$, out of which approximately $2.5 \mathrm{~GB}$ of data are extracted after the preprocessing step. The data consist of 6490 wafers, spread across 286 lots. The die level test has 14 bins. Each machine has on average 172 lots going through it. The length of measurements and operations for each lot is approximately 570, and there are 1482 (machine, measurements, process number) combinations.

\subsubsection{Defect Detection}

Since the clustering algorithm uses $k$-means as part of the solution, we first perform experiments on the actual production run data to determine the number of points to be maintained for the hierarchical clustering. We execute our algorithm 10 times and obtain the average. Table 1.1 presents the average running time of our algorithm by varying the number of points reduced. We note that $10 \%$ is a reasonable value for number of points to be kept. Although we can gain better speed using only $5 \%$, we use $10 \%$ to maintain the accuracy of the heirarchical clustering.

We also examine the effect of different distance metrics. We applied the Manhattan distance as well as the Kullback-Leibler distance (Kullback and Leibler, 1951) and its variants such as Jeffrey's divergence and Jessen's difference (Taneja, 1995), along with the resistor-average distance (Johnson and Sinanović, 2001). The results can be seen in Table 1.2. The stopping criteria used are the separateness of the clusters - that is, if the intercluster distance is at 
TABLE 1.2. Distance Metrics

\begin{tabular}{lc}
\hline Distance Metric & $\begin{array}{c}\text { Number of } \\
\text { Clusters Obtained }\end{array}$ \\
\hline Kullback-Leibler distance & 2 \\
Jeffrey's divergence & 326 \\
Resistor-average distance & 261 \\
Jesse's difference & 256 \\
Euclidean & 6 \\
Manhattan & 3 \\
\hline
\end{tabular}

least as large as the intracluster distance. As we can see, the Kullback-Leibler distance and its variants are not suitable for this application because they result in either too few or too many clusters. Manhattan and Euclidean distance performs similarly. The Euclidean distance is used for the rest of the experiments.

Due to the randomness in choosing the initial start points in our clustering algorithms, we execute our program a number of times and observe the trends of the clustering results. We find that, on average, there are five to six clusters produced, with three clusters having large enough members ( $>30$ wafers), while the rest are most likely outliers, containing only one to two wafers per cluster. Therefore, we only use the three large clusters and omit the smaller clusters. The reason for this phenomenon can be best explained using Figure 1.7, where we plot the cumulative distribution of the wafer's yield. We observe that there are roughly three prominent areas: those above 0.8 , those below 0.1 , and those between 0.55 and 0.8 . There is a concentration of points above 0.8 with a peak at around 0.92 , which corresponds to those wafers with high yield. As for the defect clusters, there are two regions where the points are concentrated: those below 0.1 , which are referred as zero yield wafers (wafers with very low yield and thus considered to have zero yield), and those wafers with 0.55 to 0.8 yield. This agrees with the three clusters that we found.

The bin distribution of clusters from one of our runs is shown in Figure 1.8. The first three bins are those with 1 or 2 members, while the last three are the larger clusters. Cluster 5 is the high-yield cluster, as can be seen from its high proportion of Bin 1.

We verify our results with the engineers. They point out that the problem they are interested in is represented by one of the cluster-in particular, the cluster with medium yield. This cluster contains three bins where the problem is prominent. They are Bins 3, 5, and 9. The zoomed-in version of these bins are shown in Figure 1.8. Bin 3 is most affected by the problem, and Cluster 6 has a significant portion of its die count fall into this bin. Cluster 3 , which has only one member, has a profile similar to that of Cluster 6 on these bins. However, there is a big difference in Bin 4 that leads to it not being combined into Cluster 6. Cluster 4 represents the zero-yield wafers as can be seen from its distribution in Bin 15, which is also the special bin to cater for untested dies. Overall, our 


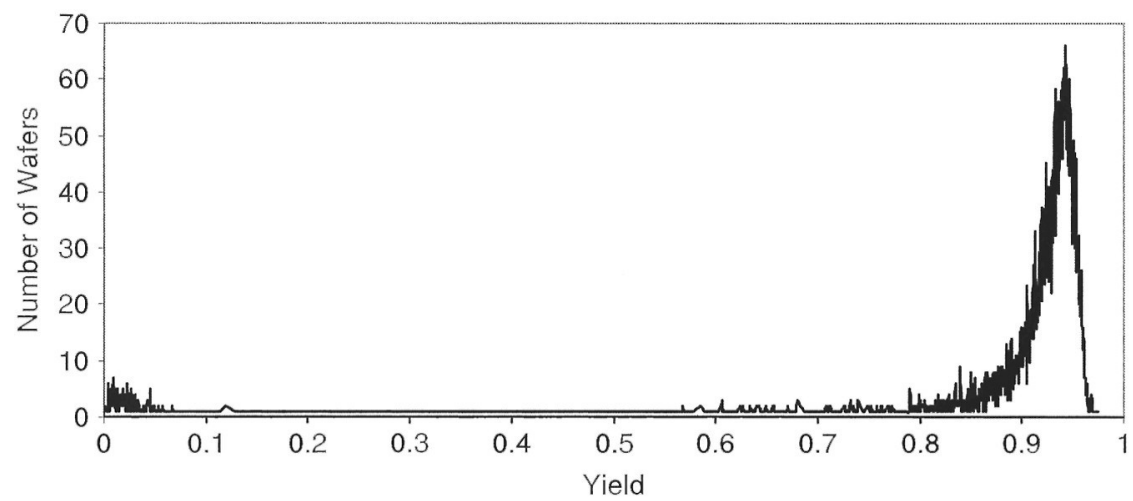

Figure 1.7. Yield plot of a wafer.
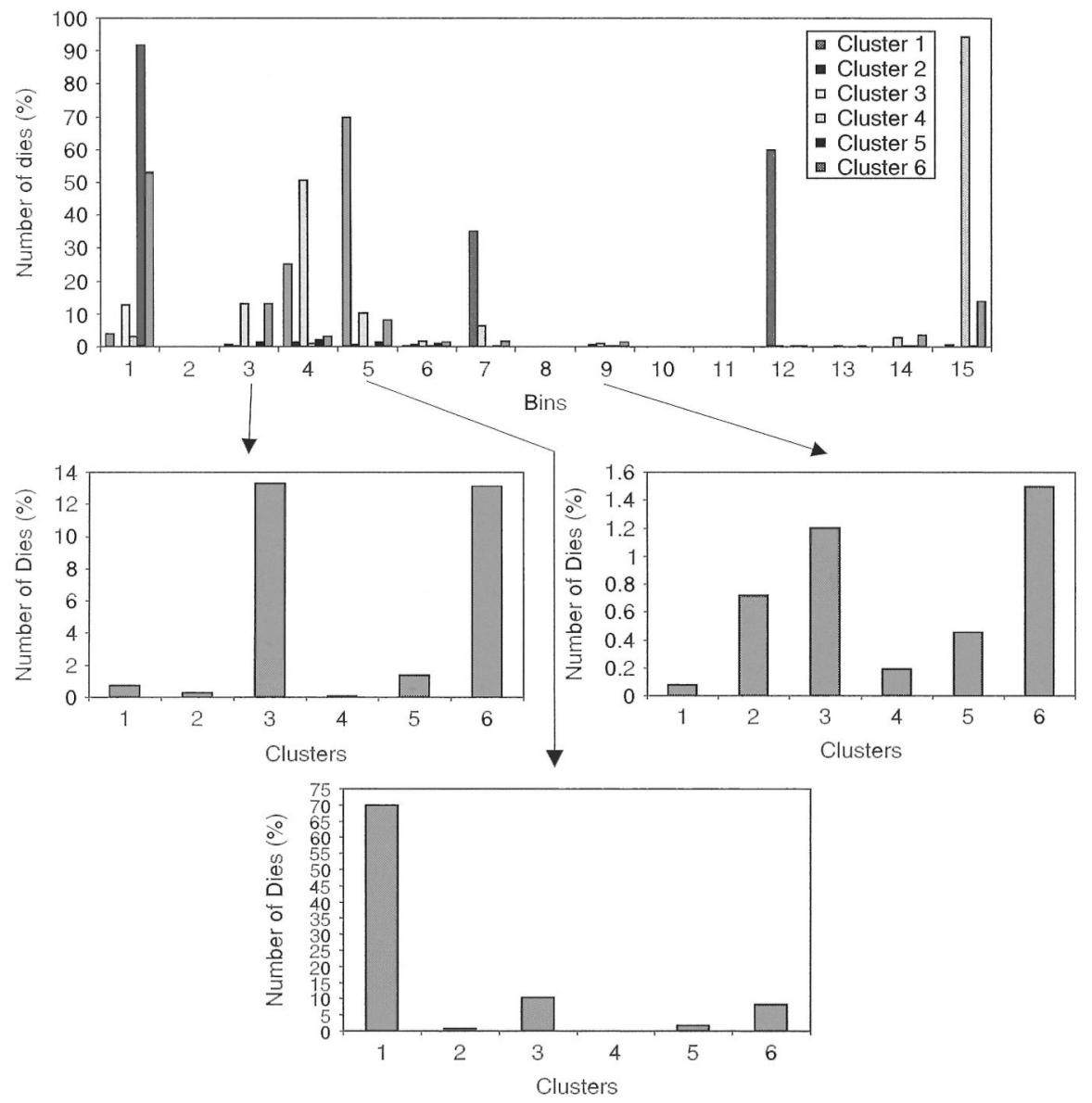

Figure 1.8. Bin profiles for all clusters. 
defect identification has succeeded in identifying problematic wafers from their bin profiles as verified by the engineers.

In addition, we observe that it is important for the clustering algorithm to introduce the bias. Otherwise, the representatives will all come from the highyield range, and we will miss the problem signature that the algorithm will consider as an outlier. This approach addresses the issue that we need to maintain sensitivity, yet at the same time efficiently zoom in to the right points.

\subsubsection{Isolating Defect Source}

Next, we focus our attention to determine the source of defects from the operational data. Since the problem has been found to be correlated to Cluster 6 , we will describe our findings that are related to this cluster.

We use the filtering algorithm as discussed in Section 1.4.3, and we present the top $30 \%$ (machine, process number) list obtained for Cluster 6 to the engineers. We choose $30 \%$ since the list will include certain machines that are needed for several operations. The algorithm terminates when we have about 5 to 10 machines in the list.

An independent inspection that was carried out by the engineers confirmed that the source of the problem was due to an over-etching in the identified machine. In particular, one of the etch machines has a problem of over-etching the wafers throughout the end of February and March. Figure 1.9a shows the nitride residue

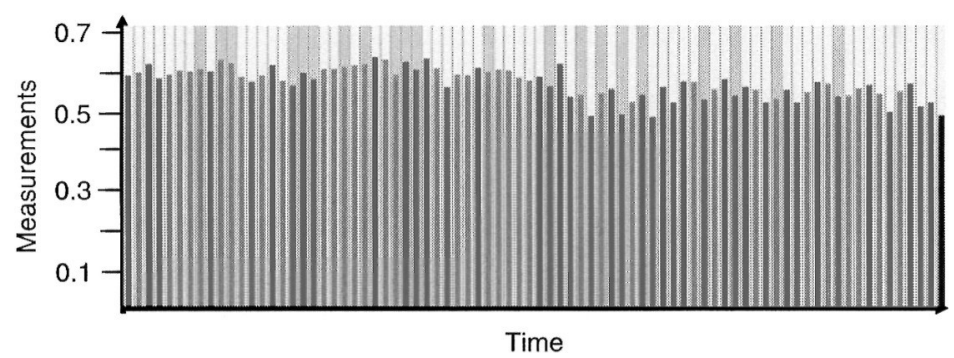

(a)

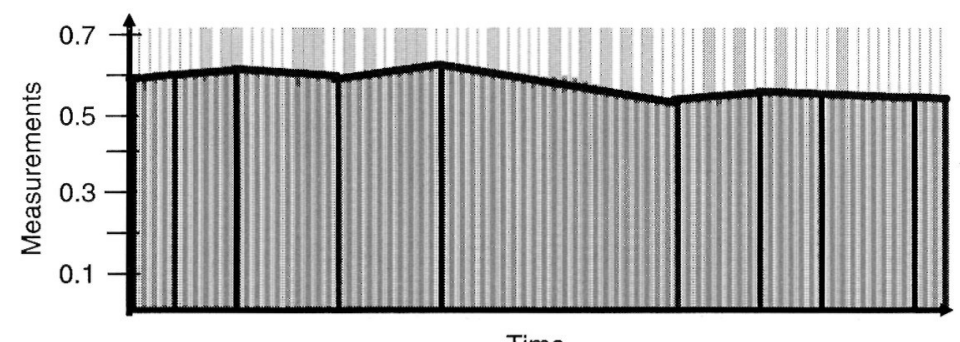

Time

(b)

Figure 1.9. (a) Original thickness measure after etch process. (b) Smoothened thickness measure after etch process. 


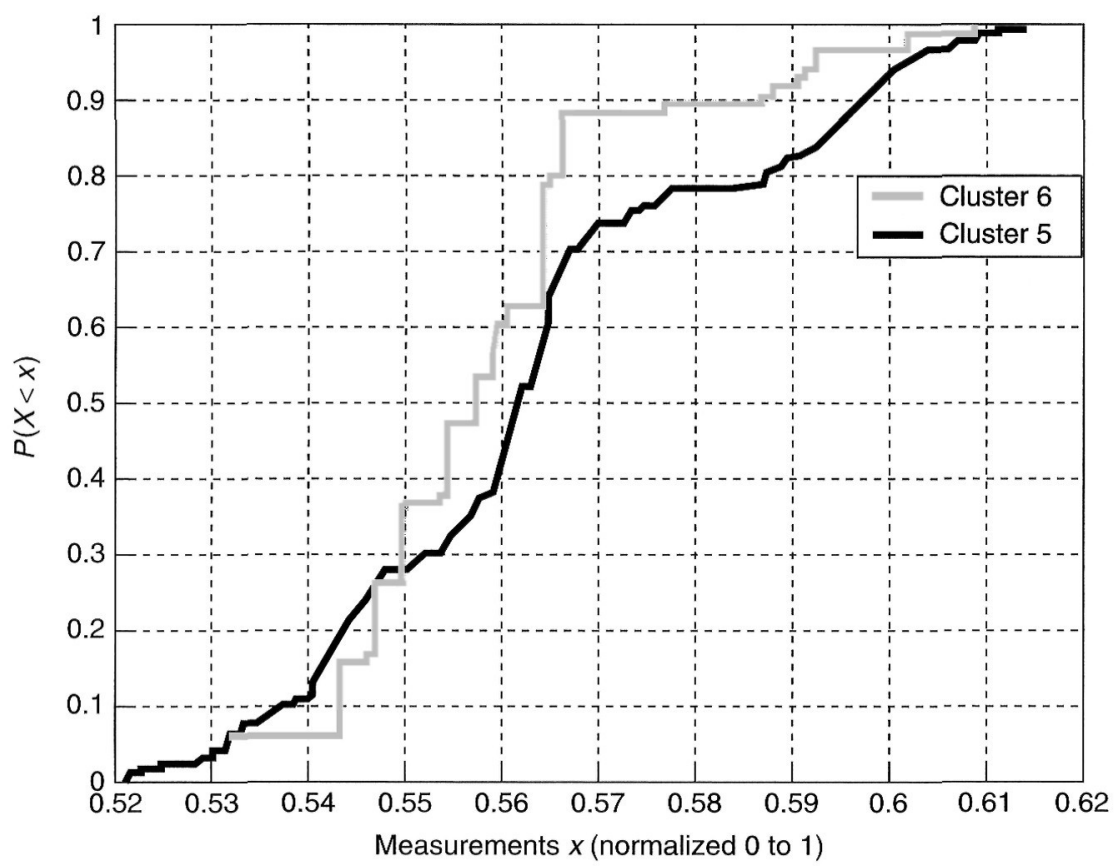

Figure 1.10. CDF of Clusters 5 and 6.

measurements of wafers, obtained after the etching process. The first half of the graph presents nitride measurements before the over-etching problem, and the second half of the graph presents nitride measurements after the over-etching problem occurred. The dark and light bars represent the change points detected. As we can see from Figure 1.9b, there is an obvious downward trend for the second half of the graph. This corresponds to thinner nitride residue observed after the etch process.

Figure 1.10 shows the difference in measurements of one of the listed machines for Cluster 5 (the good cluster) and Cluster 6 (the problematic cluster), respectively. It depicts the cumulative distribution function, $\operatorname{cdf}(x)$, which is the probability to observe a value less than $x$, that is, $P(X<x)$. We observe that for the same value of $x$ (around 0.56 to 0.57 ), the $\operatorname{cdf}(x)$ for Cluster 6 is much higher than that of Cluster 5. This relates to the fact that Cluster 6 has a higher probability of having a value less than 0.57 than does Cluster 5 . In other words, this machine is likely to be the source of the defects.

This study indicates that the filtering algorithm is able to (a) reduce the number of irrelevant features based on the variability of machine's measurements and (b) differentiate high-yield clusters from low-yield clusters. Furthermore, although we reduce the number of features used to $30 \%$ of the original number, we are able to identify the real cause of the defect. This filtering solution addresses the problem of small sample size. The weighting scheme bridges the data gap, and at 
the same it time considers as much data as possible to (a) maximize the number of data points that we can analyze and (b) derive a valid hypothesis.

\subsection{CONCLUSION}

In this chapter we have presented a framework to unify the various data-mining solutions for wafer manufacturing data. We have seen the complex wafer manufacturing process, and it is a challenge to mine wafer fabrication data: The small sample of operational data obtained from the fabrication process is highdimensional, while the large amounts of final test data from the output of the fabrication process are low-dimensional.

We have designed a bin-profile-based defect detection method to discover problematic wafers. This method utilizes a two-phase clustering algorithm to efficiently group the defect wafers. This algorithm provides a speed-up over the traditional hierarchical agglomerative clustering at the cost of approximation for lower levels of the dendogram. We have also presented an area-sensitive sampling method to select initial starting points which introduces bias as to which local minima the $k$-means should reach in order to group points together. We have designed and implemented an efficient defect signature detection algorithm from the bin profile of the wafers.

We have also developed a domain-knowledge-aided feature filtering that is able to reduce $70 \%$ of the features, differentiate high-yield clusters from low-yield clusters, and yet retain the actual cause of problematic wafers. The experiment results indicate that data-mining techniques have the potential to help discover defective wafers as well as to identify the source of the problem. We have shown that the proposed approach is able to produce results much faster than the existing manual verification process.

Future works includes building the other components of the framework, as well as incorporating existing solutions such as combining bin profile defect detection with spatial signature of the wafers.

\section{REFERENCES}

Agrawal, R., and Srikant, R., Fast algorithms for mining association rules, in Proceedings of the 20th VLDB Conference, 1994.

Bertino, E., Catania, B., and Caglio, E., Applying data mining techniques to wafer manufacturing, PKDD, 1704 41-50 (1999).

Bradley, P. S., Fayyad, U., and Reina, C., Initialization of iterative refinement clustering algorithms, in Proceedings of ACM SIGKDD, $1998 \mathrm{a}$.

Bradley, P. S., Fayyad, U., and Reina, C., Scaling Clustering Algorithms to Large Databases, in Proceedings of ACM SIGKDD, 1998b.

Dash, M., Efficient Clustering for Knowledge Discovery in Large High-Dimensional Databases, Ph.D. Thesis, School of Computing, NUS, 2002.

Dash, M., and Liu, H., Feature selection for classification, Intelligent Data Analysis 1, 1997. 
Dash, M., Tan, K.-L., and Liu, H., Efficient yet accurate clustering, in Proceedings of the Ist IEEE International Conference on Data Mining, 2001.

Devore, J. L., Probability and Statistics for Engineering and the Sciences, 4th edition, Wadsworth, Belmont, CA, 1995.

Dubes, R. C., and Jain, A. K., Algorithms for Clustering Data, Prentice-Hall, Englewood Cliffs, NJ, 1988.

Ester, M., Kriegel, H-P., Sander, J., and Xu, X. A density based algorithm for discovering clusters in large spatial databases with noise, in Proceedings of ACM SIGKDD, pp. 226-231, 1996.

Fountain, T., Dietterich, T., and Sudyka, B., Mining IC test data to optimize VLSI testing, in Proceedings of ACM SIGKDD, 2000.

François, B., and Yves, C., Improving Yield in IC Manufacturing by Statistical Analysis of a Large Database, http://www.micromagazine.com/archive/99/03/bergeret.html, 1999.

Frawley, W. J., Piatetsky-Shapiro, G., and Matheus, C., Knowledge discovery in databases: An overview, in Knowledge Discovery in Databases, AAAI Press/MIT Press, Cambridge, MA, pp. 1-30, 1991.

Gardner, M., and Bieker, J., Data mining solves tough semiconductor manufacturing problems, in Proceedings of ACM SIGKDD, 2000.

Guha, S., Rastogi, R., and Shim, K., CURE: An efficient clustering algorithm for large databases, in Proceedings of ACM SIGMOD, 1998.

Hotelling, H. Analysis of complex statistical variables into principal components, Journal of Educational Psychology 24, 417-441 (1993).

Jain, A. K., Murty, M. N., and Flynn, P. J., Data clustering: A review, ACM Computing Surveys 31(3), 264-323 (1999).

John, G. H., Kohavi, R., and Pfleger, K., Irrelevant features and the subset selection problem, in Proceedings of the Eleventh International Conference on Machine Learning, 1994.

Johnson, D. H., and Sinanović, S., Symmetrizing the Kullback-Leibler distance, IEEE Transactions on Information Theory, 2001.

Kittler, R., and Wang, W., Data mining for yield improvements, in International Conference on Modeling and Analysis of Semiconductor Manufacturing, 2000.

Kohonen, K., Self-Organizing Maps, Springer-Verlag, Berlin, 1995.

Kullback, S., and Leibler, R. A., On information and sufficiency, Annals of Math. Statistics 22, 79-86 (1951).

Kusiak, A., Decomposition in data mining: An industrial case study, IEEE Transactions on Electronic Packaging Manufacturing 23(4), 238-283 (2000).

Linde, Y., Buzo, A., and Gray, R. M., An algorithm for vector quantizer design, IEEE Transactions on Communications 28, 84-95 (1980).

Liu, B., Hsu, W., and Ma, Y., Integrating classification and association rule mining, in Proceedings of ACM SIGKDD, 1998.

Lloyd, S. P., Least squares quantization in PCM, IEEE Transactions on Information Theory $28,127-135$ (1982).

MacQueen, J., Some methods for classification and analysis of multivariate observations, in Proceedings of the 5th Berkeley Symposium on Mathematical Statistics and Probability, 1967.

Matoušek, J., On approximate geometric $k$-clustering, Discrete and Computational Geometry 24, 61-84 (2000).

Mitchell, T., Machine Learning, McGraw-Hill, New York, 1997. 
Montgomery, D., Introduction to Statistical Quality Control, John Wiley \& Sons, New York, 1991.

Povinelli, R. J., Time Series Data Mining: Identifying Temporal Patterns for Characterization and Prediction of Time Series Events, Ph.D. Dissertation, Marquette University, Milwaukee, WI, 1999.

Quadrillion Co., Semiconductor Yield Enhancement Software, http://www.quadrillion.com, 2003.

Quinlan, R., c4.5: Program for Machine Learning, Morgan Kaufmann, San Mateo, CA, 1992.

Taneja, I. J., New developments in generalized information measures, in Advances in Imaging and Electron Physics, Vol. 91, Academic Press, New York, 1995.

Tavalera, L., Dynamic feature selection in incremental hierarchical clustering, in Proceedings of European Conference on Machine Learning, 2000.

Tobin, K. W., Karnowski, T. P., Gleason, S. S., Jensen, D., and Lakhani, F., Using historical wafermap data for automated yield analysis, Journal of Vacuum Science Technology (1999).

Zhang, T., Ramakrishnan, R., and Livny, M., BIRCH: An efficient clustering algorithm for large databases, in Proceedings of ACM SIGMOD, 1996. 